\title{
Midiendo la calidad de la democracia en España
}

\author{
Roberto Tejeda Ávila
}

ANTONIA MARTínEZ (ed.), 2006

\section{Representación y calidad de la democracia en España}

Tecnos, España.

E 1 libro que se reseña a continuación tiene como referente empírico principal dos encuestas: una a los diputados del Congreso, que se realizó entre octubre y diciembre de 2004, sobre aspectos relacionados con el ejercicio de la representación política, la vida interna de los partidos y el funcionamiento de las instituciones; $y$ las opiniones de los ciudadanos que se obtuvieron del cuestionario diseñado por el proyecto de investigación "Representación y calidad de la democra- cia en España", realizado por el Centro de Investigaciones Sociológicas durante los primeros meses de 2005. Los datos han sido usados por un grupo numeroso de especialistas en ciencias políticas, básicamente, para medir la calidad de la representación política en España.

La representación se construye a partir de la interconexión constituida entre ciudadanos y políticos, los dos actores clave del proceso. Al elaborar el marco de referencia que servirá para
Roberto Tejeda Ávila: Programa de doctorado en ciencias sociales, Centro de Investigaciones y Estudios Superiores en Antropología Social-Occidente, Guadalajara, México. tejedavila@yahoo.com.mx

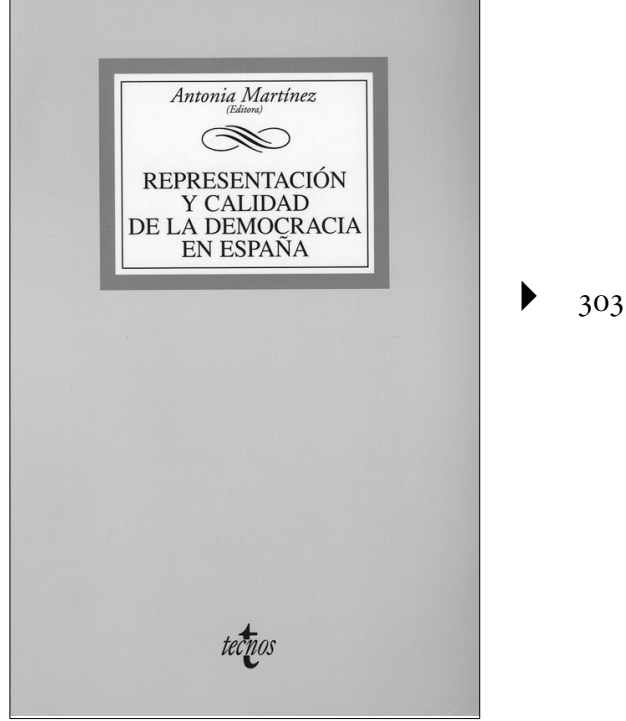

la discusión de los once capítulos que forman el libro, Antonia Martínez identifica tres escenarios de relación: la "receptividad", que se articula sobre las señales emitidas por los ciudadanos y las políticas instrumentadas, $y$ que implicaría que las decisiones de los representantes deberían responder a las aspiraciones de los ciudadanos; el segundo escenario pone en relación los mandatos con las políticas, donde 
los políticos proponen una serie de opciones a los ciudadanos - contenidas en los programas electoralesque son elegidas (o no) por los electores; el tercer escenario es el que se establece entre resultados y sanciones, denominado accountability. Este último es el mecanismo que crea los procedimientos y las condiciones por medio de las cuales los representados hacen efectivo el principio de rendición de cuentas y premian o castigan a los representantes. En este contexto, las elecciones son un procedimiento para que los ciudadanos puedan exigir responsabilidad por sus políticas a los representantes.

Aunque en los últimos años diversos estudios de opinión pública señalan que los ciudadanos están descontentos con el funcionamiento de su

\section{democracia, el análisis que realizan} Juan José García Escribano y Pilar Ortiz sobre los españoles ante la política y las instituciones de la democracia representativa demuestra que en España las encuestas reflejan con persistencia un alto nivel de apoyo ciudadano a la democracia como sistema de gobierno; asimismo, el grado de satisfacción con el funcionamiento de la democracia se mantiene en cotas elevadas. Lo que explica en mayor grado el desinterés ciudadano hacia la política y su nula o escasa participación en los asuntos públicos es la pérdida de confianza en las instituciones en general. Del análisis de distintos estudios de opinión, los profesores de la Universidad de Murcia llegan a una conclusión general: "a medida que aumenta la 'lejanía' de las instituciones — política y administrativamente- $\mathrm{o}$ son percibidas como tales por los ciudadanos, disminuye el interés que despierta la actividad desempeñada por ellas" (p. 52). Mediante su estudio, muestran que mientras que el grado de apoyo de los españoles a la democracia es bastante alto, el nivel de satisfacción con el funcionamiento de la misma es menor.

En el capítulo 3, Eva Anduiza y Robert Liñeira analizan, teniendo como referencia el sistema electoral del Congreso de los Diputados, la relación entre el sistema electoral y la calidad de la democracia. Toman en cuenta tres aspectos: los valores democráticos que se persiguen; el funcionamiento y las consecuencias de los sistemas electorales con respecto a dichos valores; y el contexto político y social en que se valora esta relación. En primer lugar abordan la definición de los principios normativos sobre los que se basa la calidad de la democracia más estrechamente vinculados con el sistema electoral: la igualdad, la representación y el control popular del gobierno. En segundo lugar analizan el caso concreto del sistema electoral del Congreso de los Diputados con relación a esos tres principios o valores. Inician su evaluación estudiando las consecuencias del sistema electoral en términos de proporcionalidad, fragmentación del sistema de partidos y gobernabilidad. Estos investigadores de la Universidad Autónoma de Barcelona se proponen no sólo evaluar el funcionamiento del sistema electoral en términos de su impacto para la calidad de la democracia, sino valorar además posibles propuestas de mejoría.

Ya se ha dicho que la representación política se construye a partir de la re- lación que se establece entre ciudadanos y políticos. Para Ismael Crespo y Antonia Martínez, este esquema relacional se configura a partir de un doble escenario: la receptividad, que implica que las políticas llevadas a cabo por los representantes han de ser sensibles a las aspiraciones ciudadanas; $y$ la accountability, rendición de cuentas que se establece entre resultados y sanciones, y que constituye el mecanismo por medio del cual se crean los procedimientos y las condiciones que servirán para que los representados evalúen las actuaciones de sus representantes $y$, posteriormente, los premien o castiguen por medio del voto. Con relación a la receptividad, los ciudadanos españoles expresan una valoración negativa sobre la influencia que sus opiniones tienen en las decisiones de sus representantes. Además, la mayoría de los encuestados en 2005 considera necesario generar nuevos mecanismos en la democracia para que la población participe más en el proceso de toma de decisiones.

El objetivo de Mónica Méndez es analizar desde una perspectiva empírica la forma en que diputados y ciudadanos conciben, perciben y valoran la representación política. La premisa que guía a esta profesora de la Universidad de Murcia es que "tanto los parlamentarios como el electorado en general tienen preferencias respecto a la forma en que debe ser desarrollada la representación política por parte de los agentes que la llevan a cabo y que esas preferencias actúan a modo de 'lentes' por medio de las cuales los individuos analizan y evalúan la calidad de la representación política" 


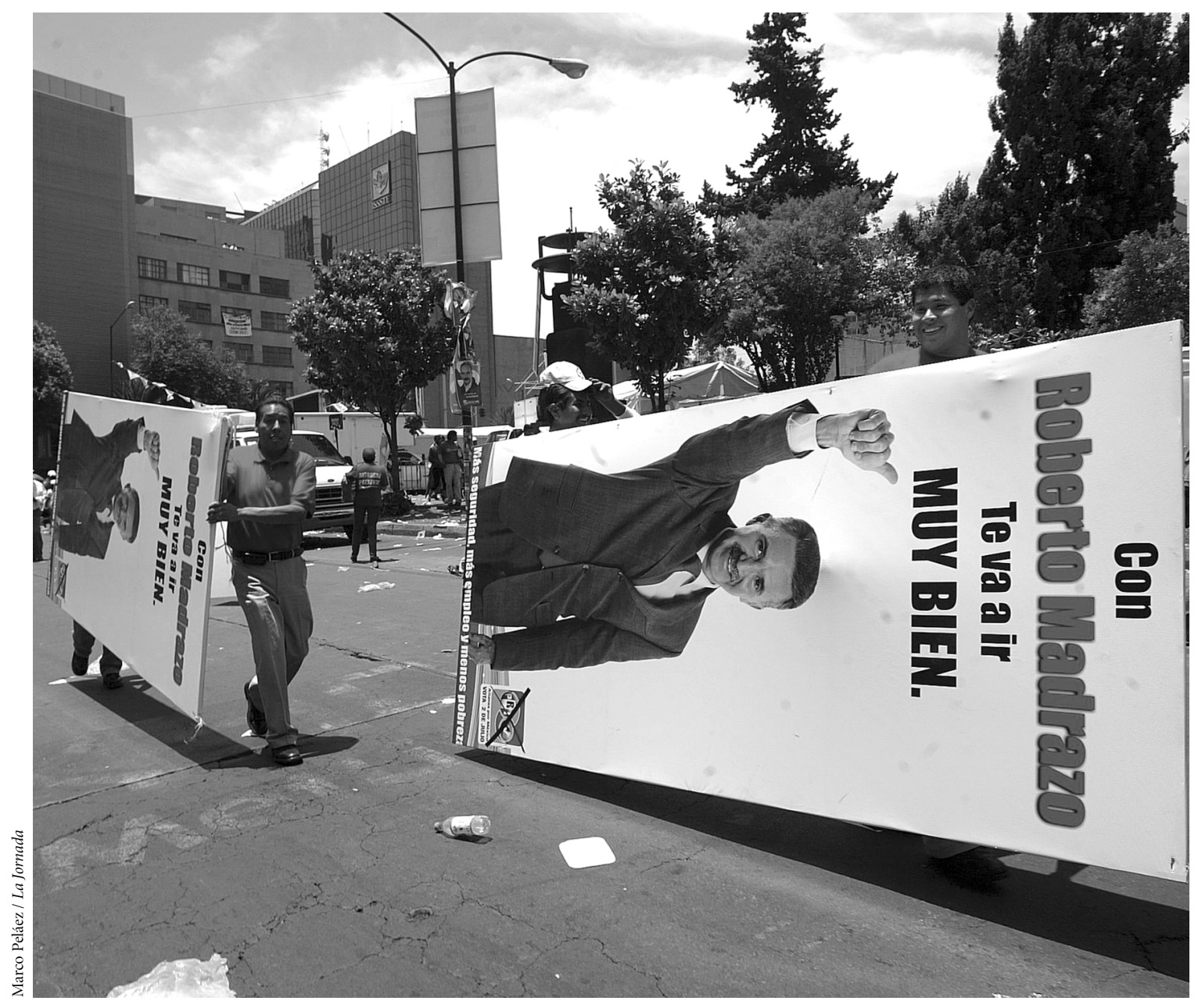

Simpatizantes de Roberto Madrazo, campaña electoral de 2006.

(p. 117). Una buena parte de los resultados de la encuesta a diputados y ciudadanos que nutren la discusión del capítulo 5 son comparados con los obtenidos en un trabajo realizado por la autora en colaboración con Antonia Martínez en 1997; se pretende comprobar si las conclusiones alcanzadas entonces en torno a la percepción de la representación política se mantienen casi una década después. La autora llega a la conclusión de que existe un alto índice de insatisfacción respecto al modo en que se lleva a cabo la representación política, así como una escasa valoración y cercanía hacia los partidos políticos.

En el capítulo 6, Mireia Grau y Mónica Méndez estudian la relación entre las características cuasifederales del Estado de las Autonomías y la dimensión territorial de la representación política. Les interesa saber cómo y hasta qué punto el entramado institucional autonómico influye en la manera en que los encuestados conciben la representación política. Revisan las principales propuestas que abordan la relación entre el federalismo y la representación política, y realizan una breve caracterización de los rasgos de la representación política territorial en España. Analizan los datos de las encuestas realizadas a ciudadanos y diputados con el propósito de explorar empíricamente las opiniones esbozadas respecto 
a la medida en que diputados y partidos representan intereses territoriales. Esperan conocer "si existe una correspondencia entre los incentivos presentes en el Estado de las Autonomías que contribuyen a forjar el papel de los partidos como representantes de los intereses territoriales, y las percepciones que los ciudadanos y los propios diputados tienen del papel de los diputados como representantes de tales intereses" (p. 146). Tratan de responder a la pregunta de qué factores intervienen en que los partidos políticos territoriales y de ámbito estatal se erijan en representantes de los intereses territoriales en un sistema federal. Observan que, para el caso español, "el escaso desarrollo de instituciones de representación territorial en el ámbito estatal hace que sean los partidos políticos los que asuman la defensa de los intereses autonómicos" (p. 162). Concluyen que las opiniones de diputados y ciudadanos ponen de manifiesto las tensiones existentes en relación con la uniformidad-diversidad de las políticas que deben defender los partidos en el conjunto del Estado. Los desafíos de futuro del Estado autonómico se refieren a la forma de articular y canalizar esas tensiones.

La estructura institucional española contempla mecanismos de exigencia de responsabilidad jurídica del gobierno por parte del Parlamento, de control ordinario de la acción gubernamental y de rendición de cuentas ante las Cámaras. Estos mecanismos -numerosos y diversos - componen un conjunto heterogéneo en el que cada uno de ellos responde a su propia lógica y persigue objetivos diferentes. No obstante, todos se originan con el mismo propósito: el de fortalecer extraordinariamente la posición del gobierno. A partir de una primera inclusión en la Constitución española de 1978, su regulación ha sido relativamente extensa. Su uso y eficacia han dependido también de las características de los distintos escenarios políticos que se han sucedido desde las primeras elecciones democráticas. Su regulación fundamental, contenida básicamente en el Reglamento del Congreso de los Diputados, permanece vigente desde 1982: no se ha adaptado a los cambios de la competencia política, la comunicación y la vida social española experimentados desde entonces. Los intentos de reforma que se han llevado a cabo desde finales de la década de 1980 no consiguen fructificar o institucionalizar nuevas prácticas.

Enrique Guerrero se propuso abordar a fondo esta discusión en el capítulo 7. Para hacerlo, establece un balance de su rendimiento a lo largo de más de un cuarto de siglo y hace referencia a algunos de los cambios que han sido defendidos de manera más reiterada en los referidos intentos de reforma. El profesor de la Universidad Complutense de Madrid llega a la conclusión de que no se puede sostener que los mecanismos de responsabilidad jurídica parlamentaria se hayan comportado en la práctica como instrumentos del control efectivo del gobierno.

En el capítulo 8, Tania Vergé nos muestra que los partidos políticos mantienen diferentes concepciones de la representación política y construyen distintos equilibrios en su desarrollo particular del concepto. Le interesa conocer los presupuestos teóricos a partir de los cuales parte la evaluación que los partidos políticos hacen del proceso representativo, así como observar la coherencia con los cambios introducidos en sus discursos y estructura organizativa. Este análisis se realiza en un momento en que los partidos tienen que satisfacer a un electorado que no cuestiona su legitimidad, pero que muestra un alto descontento con su funcionamiento. Esta politóloga de la Universidad Pompeu Fabra se da a la tarea de descubrir el valor que otorga cada uno de los tres partidos españoles de ámbito estatal a las diferentes dimensiones de la representación política. Desarrolla en distintas etapas la confirmación de la existencia de una disputa por el concepto entre el Partido Socialista (PSOE), el Partido Popular (PP) e Izquierda Unida (IU), a partir del siguiente planteamiento: "los partidos defienden distintas concepciones de la democracia y construyen diferentes equilibrios entre las dimensiones" (p. 180). Con el fin de recoger los elementos que caracterizan a cada partido en una determinada concepción, analiza los documentos políticos aprobados por los órganos federales de los tres partidos. Finalmente, valora si los cambios que han introducido durante los años de democracia en España son coherentes con dichos planteamientos. Mediante su estudio se llega a una conclusión paradójica: la aplicación de algunas propuestas en el seno de los partidos que pretende reforzar o mejorar determinados aspectos de la representación política tiene consecuencias negativas 
sobre otros aspectos de este proceso.

En el siguiente capítulo se estudian los medios de comunicación como puentes para la representación política. Cuatro especialistas en el área de comunicación de la Universidad Complutense de Madrid y el Centro Universitario Villanueva, Madrid, abordan el análisis de la representación política desde una perspectiva triangular, tomando como base la interacción entre diputados, medios y ciudadanos. Su objetivo es explorar el papel que desempeñan los medios de comunicación en la representación política. Para hacerlo comparan las percepciones que poseen ciudadanos y diputados sobre la representación de los ciudadanos en el Congreso y en los medios de comunicación. Cabe señalar que para esta investigación no se realizaron encuestas a periodistas que permitan establecer paralelismos con las encuestas realizadas a diputados y a ciudadanos. Los autores se sirven de algunos estudios publicados sobre las actitudes profesionales de los periodistas ante la política. La investigación muestra que los ciudadanos valoran más positivamente la representación que de ellos se hace en los medios que la que se hace en el Congreso; además, casi todas las funciones de los medios están mejor valoradas por los ciudadanos que por los políticos. Lo más interesante es que "esa diferencia en la valoración que diputados y ciudadanos hacen de los medios de comunicación no deriva en un distanciamiento de ciudadanos frente a lo político" (p. 230), sino todo lo contrario. Su análisis nos muestra unos medios de comunicación que pueden estar fun- cionando como puente para la participación política.

¿Qué preferencias y comportamientos se observan en los ciudadanos y representantes políticos en relación con los mecanismos y procedimientos de participación directa de la ciudadanía en la toma de decisiones políticas? Ésta es la pregunta que guía el estudio que Fabiola Mota, profesora de ciencia política en la Universidad de Murcia, nos presenta en el capítulo 10. Indaga las posibilidades de profundización de la democracia representativa por medio de la introducción y extensión de mecanismos de participación política directa de los ciudadanos, y analiza la demanda de nuevos mecanismos de participación política por parte de ciudadanos y de representantes políticos. En pocas palabras, se exploran las posibilidades de la democracia participativa en España a partir de datos de encuestas, y la indagación refleja el siguiente resultado: "los ciudadanos que son miembros y/o participan en organizaciones culturales y artísticas, ONG, partidos políticos y sindicatos, son los que más probablemente conocen y participan en los mecanismos de intervención directa. En general, la implicación en organizaciones de carácter político (incluidas las $\mathrm{ONG}$ ) favorece la implicación en mecanismos de democracia participativa" (p. 246).

En el último capítulo del libro se formulan dimensiones de análisis útiles para el estudio detallado sobre los mecanismos de participación asociativa que hoy pautan el desarrollo de las políticas sociales en España. Analizando algunas experiencias concretas de participación en instancias de diag- nóstico y formulación, así como de aplicación de políticas sociales de infancia y de juventud, Cecilia Rossel, quien trabaja en el Instituto Universitario de Investigación Ortega y Gasset, intenta plantear las principales claves en torno a la participación de las entidades sociales en diferentes fases de las políticas y sus implicaciones en el debate sobre la representación política y sobre la reforma del Estado en España. Su estudio concluye que las políticas de atención a la infancia y la juventud ocupan un lugar privilegiado dentro de los ámbitos en los que las entidades sociales han tenido más presencia durante los últimos años, porque combinan el traspaso de recursos para la cogestión de servicios con la búsqueda de espacios que intentan dar a las asociaciones un lugar en el diseño de las políticas. El análisis se basa en las opiniones y percepciones de personas que, desde la Administración y desde las organizaciones de la sociedad civil, han estado involucradas de manera directa en estas instancias de participación asociativa.

Con los resultados que refleja el libro en su conjunto, los autores reseñados se suman al esfuerzo de diversos especialistas - que rebasa las fronteras nacionales- que estudian la calidad de las democracias realmente existentes y que se han dado a la tarea de encontrar referentes empíricos, cuyo conocimiento y manejo contribuya a la construcción de sociedades más democráticas. Bienvenido sea, pues, el aporte cuantitativo de los científicos españoles. 